\title{
Nonlinear Fractional $q$-Difference Equation with Fractional Hadamard and Quantum Integral Nonlocal Conditions
}

\author{
Athasit Wongcharoen, ${ }^{1}$ Arisa Thatsatian, ${ }^{2}$ Sotiris K. Ntouyas $\mathbb{D}^{3,},{ }^{3,4}$ and Jessada Tariboon ${ }^{5}$ \\ ${ }^{1}$ Department of Mechanical Engineering Technology, College of Industrial Engineering Technology, King Mongkut's University of \\ Technology North Bangkok, Bangkok 10800, Thailand \\ ${ }^{2}$ Department of General Educations, Merchant Marine Training Centre, Bang Nang Kreng, Sukhumvit Rd., \\ Samutprakarn 10270, Thailand \\ ${ }^{3}$ Department of Mathematics, University of Ioannina, 45110 Ioannina, Greece \\ ${ }^{4}$ Nonlinear Analysis and Applied Mathematics (NAAM)-Research Group, Department of Mathematics, Faculty of Science, \\ King Abdulaziz University, P.O. Box 80203, Jeddah 21589, Saudi Arabia \\ ${ }^{5}$ Intelligent and Nonlinear Dynamic Innovations, Department of Mathematics, Faculty of Applied Science, King Mongkut's University \\ of Technology North Bangkok, Bangkok 10800, Thailand
}

Correspondence should be addressed to Jessada Tariboon; jessada.t@sci.kmutnb.ac.th

Received 3 July 2020; Revised 16 September 2020; Accepted 22 September 2020; Published 5 October 2020

Academic Editor: Serkan Araci

Copyright (C) 2020 Athasit Wongcharoen et al. This is an open access article distributed under the Creative Commons Attribution License, which permits unrestricted use, distribution, and reproduction in any medium, provided the original work is properly cited.

In this paper, we establish existence and uniqueness results for a boundary value problem consisting by a nonlinear fractional $q$-difference equation subject to a new type of boundary condition, combining the fractional Hadamard and quantum integrals. Our analysis is based on Banach's fixed point theorem, a fixed point theorem for nonlinear contractions, Krasnosel'ski i's fixed point theorem, and Leray-Schauder nonlinear alternative. Examples are given to illustrate our results.

\section{Introduction}

The aim of this paper is to investigate the existence and uniqueness of solutions for a nonlinear fractional $q$-difference equation subject to fractional Hadamard and quantum integral condition of the form:

$$
\left(\begin{array}{l}
D_{q}^{\alpha} x(t)=f(t, x(t)), 1<\alpha \leq 2, t \in(0, T), \\
x(0)=0, \sum_{i=1}^{n} \gamma_{i} I_{p_{i}}^{\mu_{i}} x\left(\xi_{i}\right)=\sum_{j=1}^{m} \beta_{j} J^{\sigma_{j}} x\left(\eta_{j}\right),
\end{array}\right.
$$

where $D_{q}^{\alpha}$ is the fractional $q$-derivative of order $\alpha$, with a quantum number $q \in(0,1), f:[0, T] \times \mathbb{R} \longrightarrow \mathbb{R}$ is a nonlinear continuous function, $I_{p_{i}}^{\mu_{i}}$ denotes the fractional quantum integral of order $\mu_{i}>0$, with quantum number $0<p_{i}<1$,
$J^{\sigma_{j}}$ is the Hadamard fractional integral of order $\sigma_{j}>0, \gamma_{i}$ and $\beta_{j}$ are given constants, and $\xi_{i}, \eta_{j} \in(0, T)$ are fixed points, for $i=1, \cdots, n$ and $j=1, \cdots, m$.

The subject of fractional differential equations has recently evolved into an interesting subject for many researchers due to its multiple applications in economics, engineering, physics, chemistry, signal analysis, etc. Various types of fractional derivative and integral operator were studied: Riemann-Liouville, conformable fractional integral operators, Caputo, Hadamard, Erdelyi-Kober, GrünwaldLetnikov, Marchaud, and Riesz are just a few to name. The Hadamard-type fractional derivative differs from the preceding ones in the sense that the kernel of the integral and derivative contain logarithmic function of arbitrary exponent. Details and properties of Hadamard fractional derivatives and integrals can be found in Kilbas et al. [1]. Recently, there were some results on Hadamard-type 
fractional differential equations, see [2-11] and references cited therein.

Nonlinear fractional $q$-difference equations appear in the mathematical modeling of many phenomena in engineering and science and have attracted much attention by many researchers, see for example [12-21] and references therein.

In the present paper, the novelty lies in the fact that we combine in boundary conditions both Hadamard and quantum integrals. To the best of our knowledge, this type of boundary condition appears for the first time in the literature. It is important to notice that we are combining in our work, fractional calculus, and quantum calculus. The key tool for this combination is the Property 2.25 of [1].

Some special cases of the second condition of (1) can be seen by reducing $m=n=1$ as

$$
\begin{gathered}
\frac{\gamma_{1}}{\Gamma_{p_{1}}\left(\mu_{1}\right)} \int_{0^{+}}^{\xi_{1}}\left(\xi_{1}-p_{1} s\right)_{p_{1}}^{\left(\mu_{1}-1\right)} x(s) d_{p_{1}} s \\
=\frac{\beta_{1}}{\Gamma\left(\sigma_{1}\right)} \int_{0^{+}}^{\eta_{1}}\left(\log \frac{\eta_{1}}{s}\right)^{\sigma_{1}-1} \frac{x(s)}{s} d s,
\end{gathered}
$$

which is mixed quantum and Hadamard calculus. If $p_{1}=1$, then we have

$$
\begin{aligned}
& \frac{\gamma_{1}}{\Gamma\left(\mu_{1}\right)} \int_{0^{+}}^{\xi_{1}}\left(\xi_{1}-s\right)^{\mu_{1}-1} x(s) d s \\
& \quad=\frac{\beta_{1}}{\Gamma\left(\sigma_{1}\right)} \int_{0^{+}}^{\eta_{1}}\left(\log \frac{\eta_{1}}{s}\right)^{\sigma_{1}-1} \frac{x(s)}{s} d s,
\end{aligned}
$$

which is also mixed Riemann-Liouville and Hadamard fractional integral condition. If $\mu_{1}=\sigma_{1}=1$, we have integral condition of the form:

$$
\gamma_{1} \int_{0^{+}}^{\xi_{1}} x(s) d s=\beta_{1} \int_{0^{+}}^{\eta_{1}} \frac{x(s)}{s} d s
$$

which is a variety used in physical boundary value problems.

We establish existence and uniqueness results by using standard fixed point theorems. We prove two existence and uniqueness results with the help of the Banach contraction mapping principle and a fixed point theorem on nonlinear contractions due to Boyd and Wong. Moreover, we prove two existence results, one via Leray-Schauder nonlinear alternative and another one via Krasnosel'ski i's fixed point theorem.

The paper is organized as follows: in Section 2, we recall some preliminary facts that we need in the sequel. In Section 3 , we prove our main results. Some examples to illustrate our results are presented in Section 4.

\section{Preliminaries}

To present the preliminary, we suggest the basic quantum calculus in the book of Kac and Cheung [22], fractional quantum calculus in [23-25], and the Hadamard fractional calculus in [1]. Let a fixed constant $q \in(0,1)$ be a quantum number. The $q$-number is defined by

$$
[a]_{q}=\frac{1-q^{a}}{1-q}, a \in \mathbb{R} .
$$

For example, $[3]_{q}=1+q+q^{2}$. The $q$-power function for any $a, b \in \mathbb{R}, a \neq 0$, is defined as

$$
(a-b)_{q}^{(\gamma)}=a^{\gamma} \prod_{i=0}^{\infty} \frac{1-(b / a) q^{i}}{1-(b / a) q^{\gamma+i}}
$$

If $\gamma=k \in \mathbb{N}_{0}=\{0,1,2, \cdots\}$, then $(a-b)_{q}^{(k)}=\prod_{i=0}^{k-1}(a-b$ $\left.q^{i}\right)$ and $(a-b)_{q}^{(0)}:=1$. For example, $(a-b)_{q}^{(3)}=(a-b)(a-q$ $b)\left(a-q^{2} b\right)$. The notation of $q$-power function is appeared in kernels of fractional $q$-calculus as Definitions 1 and 2 . Now, the q-gamma function $\Gamma_{q}(t)$ is defined by

$$
\Gamma_{q}(t)=\frac{(1-q)_{q}^{(t-1)}}{(1-q)^{t-1}}, \quad \text { for } \quad t \in \mathbb{R} \backslash\{0,-1,-2, \cdots\}
$$

Now, we observe that $\Gamma_{q}(t+1)=[t]_{q} \Gamma_{q}(t)$. Next, we discuss about the $q$-derivative of a function $f:[0, \infty) \longrightarrow \mathbb{R}$ which is defined by

$$
D_{q} f(t)=\frac{f(t)-f(q t)}{(1-q) t}, \quad t \neq 0 \text {, and } D_{\mathrm{q}} f(0)=\lim _{t \rightarrow 0} D_{q} f(t) .
$$

If $f^{\prime}(t)$ exists, then $\lim _{q \rightarrow 1} D_{q} f(t)=f^{\prime}(t)$. The $q$-integral formula can be presented as

$$
\left(I_{q} f\right)(t)=\int_{0}^{t} f(s) d_{q} s=t(1-q) \sum_{n=0}^{\infty} q^{n} f\left(t q^{n}\right), t \in[0, \infty) .
$$
is

The higher order of $q$-derivative and $q$-integral operators

$$
\begin{aligned}
\left(D_{q}^{k} h\right)(t) & =D_{q}\left(D_{q}^{k-1} f\right)(t) \text { and }\left(I_{q}^{k} h\right)(t) \\
& =I_{q}\left(I_{q}^{k-1} f\right)(t), k \in \mathbb{N},
\end{aligned}
$$

with $\left(D_{q}^{0} f\right)(t)=f(t)$ and $\left(I_{q}^{0} f\right)(t)=f(t)$. Next, the fundamental theorem of calculus for operators $D_{q}$ and $I_{q}$ can be stated as formulas

$$
\left(D_{q} I_{q} f\right)(t)=f(t)
$$

and if $f$ is continuous at the point $t=0$, then

$$
\left(I_{q} D_{q} f\right)(t)=f(t)-f(0) .
$$


Let us give the definitions of fractional quantum calculus of the Riemann-Liouville type fractional derivative and also integral operators.

Definition 1 [24]. Let a constant $\alpha \geq 0$ and $f$ be the function on $[0, \infty)$. The Riemann-Liouville fractional $q$-integral of $f$ order $\alpha$ is defined by

$$
\begin{aligned}
\left(I_{q}^{\alpha} f\right)(t)=\frac{1}{\Gamma_{q}(\alpha)} \int_{0^{+}}^{t}(t-q s)_{q}^{(\alpha-1)} f(s) d_{q} s, & \\
& \alpha>0, \quad t \in(0, \infty),
\end{aligned}
$$

and $\left(I_{q}^{0} f\right)(t)=f(t)$

Definition 2 [24]. The Riemann-Liouville fractional $q$-derivative of order $\alpha \geq 0$ of a function $f:[0, \infty) \longrightarrow \mathbb{R}$ is given by

$$
\begin{aligned}
\left(D_{q}^{\alpha} f\right)(t)= & \left(D_{q}^{n} I_{q}^{n-\alpha} f\right)(t)=\frac{1}{\Gamma_{q}(n-\alpha)} D_{q}^{n} \int_{0^{+}}^{t} \\
& \cdot(t-q s)_{q}^{(n-\alpha-1)} f(s) d_{q} s, \quad \alpha>0,
\end{aligned}
$$

and $\left(D_{q}^{0} f\right)(t)=f(t)$, where $n$ is the smallest integer greater than or equal to $\alpha$.

Now, for $t, s>0$, the $q$-beta function is presented by

$$
B_{q}(t, s)=\int_{0^{+}}^{1} u^{(t-1)}(1-q u)_{q}^{(s-1)} d_{q} u,
$$

which is related to the $q$-gamma function by

$$
B_{q}(t, s)=\frac{\Gamma_{q}(t) \Gamma_{q}(s)}{\Gamma_{q}(t+s)} .
$$

The fundamental formulas for fractional quantum calculus are in the following lemma.

Lemma $3[24,26]$. Let $\alpha, \beta \geq 0, n$ be a positive integer and $f$ be a function defined in $[0, \infty)$. Then, the following formulas hold

$$
\begin{aligned}
& \left(I_{q}^{\beta} I_{q}^{\alpha} f\right)(t)=\left(I_{q}^{\alpha+\beta} f\right)(t), \\
& \left(D_{q}^{\alpha} I_{q}^{\alpha} f\right)(t)=f(t), \\
& \left(I_{q}^{\beta} D_{q}^{n} f\right)(t)=\left(D_{q}^{n} I_{q}^{\beta} f\right)(t)-\sum_{k=0}^{n-1} \frac{t^{\beta-n+k}}{\Gamma_{q}(\beta+k-n+1)}\left(D_{q}^{k} f\right)(0) .
\end{aligned}
$$

The fractional $q$-integration of the two deferent quantum numbers is given by lemma.
Lemma 4 [27]. Let constants $\alpha, \beta>0$ and $0<p, q<1$ be quantum numbers. Then, for $\eta \in \mathbb{R}_{+}$, we have

$$
I_{p}^{\alpha} I_{q}^{\beta}(1)(\eta)=\frac{\Gamma_{p}(\beta+1)}{\Gamma_{p}(\alpha+\beta+1) \Gamma_{q}(\beta+1)} \eta^{\alpha+\beta} .
$$

The Hadamard fractional calculus is the subject of fractional derivative and integral which have logarithm kernels inside the singular integral formulas as in the definitions.

Definition 5 [1]. The Hadamard derivative of fractional order $\alpha$ for a function $f:[0, \infty) \longrightarrow \mathbb{R}$ is defined as

$$
\begin{aligned}
{ }^{H} D^{\alpha} f(t)= & \frac{1}{\Gamma(n-\alpha)}\left(t \frac{d}{d t}\right)^{n} \int_{0^{+}}^{t} \\
& \cdot\left(\log \frac{t}{s}\right)^{n-\alpha-1} \frac{f(s)}{s} d s, n=[\alpha]+1,
\end{aligned}
$$

where the notation $[\alpha]$ denotes the integer part of the real number $\alpha, \log (\cdot)=\log _{e}(\cdot)$, and $\Gamma$ is the usual Gamma function.

Definition 6 [1]. The Hadamard fractional integral of order $\alpha$ for a function $f:[0, \infty) \longrightarrow \mathbb{R}$ is defined by

$$
J^{\alpha} f(t)=\frac{1}{\Gamma(\alpha)} \int_{0^{+}}^{t}\left(\log \frac{t}{s}\right)^{\alpha-1} \frac{f(s)}{s} d s, \alpha>0,
$$

provided the integral in right hand side exists.

The key tool for combining the two type of fractional calculus in our work is the following lemma.

Lemma 7 ([1], Property 2.25). Let $\alpha>0$ and $\beta>0$. The following formulas hold

$$
J^{\alpha} t^{\beta}=\beta^{-\alpha} t^{\beta} \text { and } d^{H} D^{\alpha} t^{\beta}=\beta^{\alpha} t^{\beta} .
$$

To accomplish our main purpose, we will use the fixed point theory for considering an operator equation $x=Q \mathbb{Q} x$. For finding the operator $\mathbb{Q}$, let us see the following lemma.

Lemma 8. Suppose that the points $\xi_{i}, \eta_{j} \in[0, T]$ and the constant

$$
\Omega=\sum_{i=1}^{n} \gamma_{i} \frac{\Gamma_{p_{i}}(\alpha) \xi_{i}^{\alpha+\mu_{i}-1}}{\Gamma_{p_{i}}\left(\alpha+\mu_{i}\right)}-\sum_{j=1}^{m} \beta_{j}(\alpha-1)^{-\sigma_{j}} \eta_{j}^{\alpha-1} \neq 0,
$$

where $\alpha, \mu_{i}, p_{i}, \gamma_{i}, \sigma_{j}, \beta_{j}, i=1, \cdots, n$, and $j=1, \cdots, m$ are defined in problem (1). Then, the linear fractional q-difference equation

$$
D_{q}^{\alpha} x(t)=h(t), 0<t<T,
$$


where $h:[0, T] \longrightarrow \mathbb{R}$, and subject to mixed fractional integrals of Hadamard and quantum boundary conditions

$$
x(0)=0, \sum_{i=1}^{n} \gamma_{i} I_{p_{i}}^{\mu_{i}} x\left(\xi_{i}\right)=\sum_{j=1}^{m} \beta_{j} J^{\sigma_{j}} x\left(\eta_{j}\right)
$$

is equivalent to the linear integral equation

$$
\begin{aligned}
x(t)= & \frac{1}{\Omega}\left[\sum_{j=1}^{m} t^{\alpha-1} \beta_{j} J^{\sigma_{j}} I_{q}^{\alpha} h\left(\eta_{j}\right)-\sum_{i=1}^{n} t^{\alpha-1} \gamma_{i} I_{p_{i}}^{\mu_{i}} I_{q}^{\alpha} h\left(\xi_{i}\right)\right] \\
& +I_{q}^{\alpha} h(t) .
\end{aligned}
$$

Proof. Since $\alpha \in(1,2]$, then (23) can be written as

$$
D_{q}^{2} I_{q}^{2-\alpha} x(t)=h(t), 0<t<T
$$

Applying the fractional $q$-integral of order $\alpha$ and using Lemma 3, we obtain

$$
\begin{aligned}
I_{q}^{\alpha} D_{q}^{2} I_{q}^{2-\alpha} x(t) & =D_{q}^{2} I_{q}^{\alpha} I_{q}^{2-\alpha} x(t)-k_{1} t^{\alpha-1}-k_{2} t^{\alpha-2} \\
& =x(t)-k_{1} t^{\alpha-1}-k_{2} t^{\alpha-2}=I_{q}^{\alpha} h(t),
\end{aligned}
$$

which yields

$$
x(t)=k_{1} t^{\alpha-1}+k_{2} t^{\alpha-2}+I_{q}^{\alpha} h(t)
$$

where $k_{1}, k_{2} \in \mathbb{R}$. The first boundary condition of (24) implies that $k_{2}=0$. Then, (28) is reduced to

$$
x(t)=k_{1} t^{\alpha-1}+I_{q}^{\alpha} h(t) .
$$

Now, we apply the fractional quantum integral of Riemann-Liouville of order $\mu_{i}$ with quantum number $p_{i}$ to $(29)$ as

$$
I_{p_{i}}^{\mu_{i}} x(t)=k_{1} \frac{\Gamma_{p_{i}}(\alpha) t^{\alpha+\mu_{i}-1}}{\Gamma_{p_{i}}\left(\alpha+\mu_{i}\right)}+I_{p_{i}}^{\mu_{i}} I_{q}^{\alpha} h(t)
$$

Using Lemma 7 for taking the Hadamard fractional integral of order $\sigma_{j}$ to (29), we get

$$
J^{\sigma_{j}} x(t)=k_{1}(\alpha-1)^{-\sigma_{j}} t^{\alpha-1}+J^{\sigma_{j}} I_{q}^{\alpha} h(t) .
$$

From the second boundary condition of (24) and above two equations, it follows that

$$
\begin{aligned}
& k_{1} \sum_{i=1}^{n} \gamma_{i} \frac{\Gamma_{p_{i}}(\alpha) \xi_{i}^{\alpha+\mu_{i}-1}}{\Gamma_{p_{i}}\left(\alpha+\mu_{i}\right)}+\sum_{i=1}^{n} \gamma_{i} I_{p_{i}}^{\mu_{i}} I_{q}^{\alpha} h\left(\xi_{i}\right) \\
& \quad=k_{1} \sum_{j=1}^{m} \beta_{j}(\alpha-1)^{-\sigma_{j}} \eta_{j}^{\alpha-1}+\sum_{j=1}^{m} \beta_{j} J^{\sigma_{j}} I_{q}^{\alpha} h\left(\eta_{j}\right),
\end{aligned}
$$

and consequently

$$
k_{1}=\frac{1}{\Omega}\left[\sum_{j=1}^{m} \beta_{j} J^{\sigma_{j}} I_{q}^{\alpha} h\left(\eta_{j}\right)-\sum_{i=1}^{n} \gamma_{i} I_{p_{i}}^{\mu_{i}} I_{q}^{\alpha} h\left(\xi_{i}\right)\right] \text {, }
$$

where the nonzero constant $\Omega$ is defined by (22). Substituting the constant $k_{1}$ in (29), then, we obtain (25), which is the solution of BVP (23) and (24). The converse can be obtained by a direct computation. The proof is completed.

\section{Main Results}

At first, we denote by $\mathscr{C}=C([0, T], \mathbb{R})$ the Banach space of all continuous functions from $[0, T]$ to $\mathbb{R}$ endowed with the sup norm as $\|x\|=\sup \{|x(t)|, t \in[0, T]\}$. In view of Lemma 8 and replacing the function $h$ by $f(t, x(t))$, we define the operator $\mathbb{Q}: \mathscr{C} \longrightarrow \mathscr{C}$ by

$$
\begin{aligned}
Q x(t)= & \frac{t^{\alpha-1}}{\Omega}\left[\sum_{j=1}^{m} \beta_{j} J^{\sigma_{j}} I_{q}^{\alpha} f_{x}\left(\eta_{j}\right)-\sum_{i=1}^{n} \gamma_{i} I_{p_{i}}^{\mu_{i}} I_{q}^{\alpha} f_{x}\left(\xi_{i}\right)\right] \\
& +I_{q}^{\alpha} f_{x}(t)
\end{aligned}
$$

where $I_{q}^{\alpha} f_{x}(v)$ is denoted by

$$
\begin{aligned}
I_{q}^{\alpha} f_{x}(v) & =\frac{1}{\Gamma_{q}(\alpha)} \int_{0^{+}}^{v}(v-q s)_{q}^{(\alpha-1)} f(s, x(s)) d_{q} s \\
& :=g(v), \quad v \in\left\{t, \xi_{i}, \eta_{j}\right\}
\end{aligned}
$$

while $J^{\sigma_{j}} I_{q}^{\alpha} f_{x}\left(\eta_{j}\right)$ and $I_{p_{i}}^{\mu_{i}} I_{q}^{\alpha} f_{x}\left(\xi_{i}\right)$ are the Hadamard and quantum fractional integrals of a function $g$ as

$$
\begin{aligned}
J^{\sigma_{j}} I_{q}^{\alpha} f_{x}\left(\eta_{j}\right) & =\frac{1}{\Gamma\left(\sigma_{j}\right)} \int_{0^{+}}^{\eta_{j}}\left(\log \frac{\eta_{j}}{s}\right)^{\sigma_{j}-1} \frac{g(s)}{s} d s \\
I_{p_{i}}^{\mu_{i}} I_{q}^{\alpha} f_{x}\left(\xi_{i}\right) & =\frac{1}{\Gamma_{p_{i}}\left(\mu_{i}\right)} \int_{0^{+}}^{\xi_{i}}\left(\xi_{i}-p_{i} s\right)_{p_{i}}^{\left(\mu_{i}-1\right)} g(s) d_{p_{i}} s
\end{aligned}
$$

respectively. Now, we are going to prove the main results which are the existence criteria of solution for nonlocal mixed fractional integrals boundary value problem (1). The first, an existence and uniqueness result for (1), is given by using Banach's fixed point theorem.

Theorem 9. Let $f:[0, T] \times \mathbb{R} \longrightarrow \mathbb{R}$ be a nonlinear continuous function satisfying the assumption.

$\left(H_{1}\right)$ There exists a positive constant $L$ such that $\mid f(t, x)$ $-f(t, y)|\leq L| x-y \mid$, for each $t \in[0, T]$ and $x, y \in \mathbb{R}$.

If

$$
L \Phi<1
$$


where $\Phi$ is given by

$$
\begin{aligned}
\Phi= & \frac{T^{\alpha}}{\Gamma_{q}(\alpha+1)}\left[\frac{1}{T|\Omega|} \sum_{j=1}^{m}\left|\beta_{j}\right| \alpha^{-\sigma_{j}} \eta_{j}^{\alpha}\right. \\
& \left.+\frac{1}{T|\Omega|} \sum_{i=1}^{n}\left|\gamma_{i}\right| \frac{\Gamma_{p_{i}}(\alpha+1)}{\Gamma_{p_{i}}\left(\alpha+\mu_{i}+1\right)} \xi_{i}^{\alpha+\mu_{i}}+1\right],
\end{aligned}
$$

then the boundary value problem (1) has a unique solution on $[0, T]$.

Proof. The result allows from the operator equation $x=Q 2 x$, where the operator $Q$ is defined by (34). The Banach fixed point theorem is used to show that $\mathbb{Q}$ has a fixed point which is the unique solution of problem (1). Since the function $f$ is continuous, then, we can set $\sup \{|f(t, 0)|, t \in[0, T]\}=M$ $<\infty$. After that, we define the radius $r$ satisfying

$$
r \geq \frac{\Phi M}{1-\Phi L}
$$

of a ball $B_{r}=\{x \in C:\|x\| \leq r\}$. For any $x \in B_{r}$, we see that

$$
\begin{aligned}
|Q \mathcal{Q} x(t)| \leq & \sup _{t \in[0, T]} \frac{t^{\alpha-1}}{|\Omega|} \sum_{j=1}^{m}\left|\beta_{j}\right| J^{\sigma_{j}} I_{q}^{\alpha}\left|f_{x}\right|\left(\eta_{j}\right) \\
& \left.+\frac{t^{\alpha-1}}{|\Omega|} \sum_{i=1}^{n}\left|\gamma_{i}\right| I_{p_{i}}^{\mu_{i}} I_{q}^{\alpha}\left|f_{x}\right|\left(\xi_{i}\right)+I_{q}^{\alpha}\left|f_{x}\right|(t)\right] \\
\leq & \frac{T^{\alpha-1}}{|\Omega|} \sum_{j=1}^{m}\left|\beta_{j}\right| J^{\sigma_{j}} I_{q}^{\alpha}\left(\left|f_{x}-f_{0}\right|+\left|f_{0}\right|\right)\left(\eta_{j}\right) \\
& +I_{q}^{\alpha}\left(\left|f_{x}-f_{0}\right|+\left|f_{0}\right|\right)(T)+\frac{T^{\alpha-1}}{|\Omega|} \\
& \cdot \sum_{i=1}^{n}\left|\gamma_{i}\right| I_{p_{i}}^{\mu_{i}} I_{q}^{\alpha}\left(\left|f_{x}-f_{0}\right|+\left|f_{0}\right|\right)\left(\xi_{i}\right) \\
\leq & (L r+M)\left|\frac{T^{\alpha-1}}{|\Omega|} \sum_{j=1}^{m}\right| \beta_{j} \mid J^{\sigma_{j}} I_{q}^{\alpha}(1)\left(\eta_{j}\right) \\
& \left.+\frac{T^{\alpha-1}}{|\Omega|} \sum_{i=1}^{n}\left|\gamma_{i}\right| I_{p_{i}}^{\mu_{i}} I_{q}^{\alpha}(1)\left(\xi_{i}\right)+I_{q}^{\alpha}(1)(T)\right],
\end{aligned}
$$

in which we used the following fact:

$$
\begin{aligned}
\left|f_{x}-f_{0}\right|+\left|f_{0}\right| & =|f(v, x(v))-f(v, 0)|+|f(v, 0)| \\
& \leq L|x|+M \leq L r+M,
\end{aligned}
$$

where $v \in\left\{T, \xi_{i}, \eta_{j}\right\}$. By applying Lemmas 4 and 2.3, we have

$$
\begin{aligned}
I_{p_{i}}^{\mu_{i}} I_{q}^{\alpha}(1)\left(\xi_{i}\right) & =\frac{\Gamma_{p_{i}}(\alpha+1)}{\Gamma_{p_{i}}\left(\alpha+\mu_{i}+1\right) \Gamma_{q}(\alpha+1)} \xi_{i}^{\alpha+\mu_{i}}, \\
J^{\sigma_{j}} I_{q}^{\alpha}(1)\left(\eta_{j}\right) & =\frac{1}{\Gamma_{q}(\alpha+1)}\left(J^{\sigma_{j}} t^{\alpha}\right)\left(\eta_{j}\right)=\frac{1}{\Gamma_{q}(\alpha+1)} \alpha^{-\sigma_{j}} \eta_{j}^{\alpha} .
\end{aligned}
$$

Then, we obtain

$$
\begin{aligned}
|Q \mathcal{Q} x(t)| \leq & \frac{(L r+M) T^{\alpha}}{\Gamma_{q}(\alpha+1)}\left[\frac{1}{T|\Omega|} \sum_{j=1}^{m}\left|\beta_{j}\right| \alpha^{-\sigma_{j}} \eta_{j}^{\alpha}\right. \\
& \left.+\frac{1}{T|\Omega|} \sum_{i=1}^{n}\left|\gamma_{i}\right| \frac{\Gamma_{p_{i}}(\alpha+1)}{\Gamma_{p_{i}}\left(\alpha+\mu_{i}+1\right)} \xi_{i}^{\alpha+\mu_{i}}+1\right] \\
= & (L r+M) \Phi \leq r .
\end{aligned}
$$

From this, we conclude that $\|Q x\| \leq r$ which yields $Q B_{r} \subset B_{r}$.

Next, we will prove that the operator $\mathbb{Q}$ is a contraction. Let $x, y \in \mathscr{C}$, and for each $t \in[0, T]$, then, we have

$$
\begin{aligned}
|\mathcal{Q} x(t)-\mathcal{Q} y(t)| \leq & \frac{T^{\alpha-1}}{|\Omega|} \sum_{j=1}^{m}\left|\beta_{j}\right| J^{\sigma_{j}} I_{q}^{\alpha}\left|f_{x}-f_{y}\right|\left(\eta_{j}\right)+\frac{T^{\alpha-1}}{|\Omega|} \\
& \cdot \sum_{i=1}^{n}\left|\gamma_{i}\right| I_{p_{i}}^{\mu_{i}} I_{q}^{\alpha}\left|f_{x}-f_{y}\right|\left(\xi_{i}\right)+I_{q}^{\alpha}\left|f_{x}-f_{y}\right|(t) \\
\leq & \left(\frac{T^{\alpha-1}}{|\Omega|} \sum_{j=1}^{m}\left|\beta_{j}\right| J^{\sigma_{j}} I_{q}^{\alpha}(1)\left(\eta_{j}\right)+\frac{T^{\alpha-1}}{|\Omega|}\right. \\
& \left.\cdot \sum_{i=1}^{n}\left|\gamma_{i}\right| I_{p_{i}}^{\mu_{i}} I_{q}^{\alpha}(1)\left(\xi_{i}\right)+I_{q}^{\alpha}(1)(T)\right) \\
& \cdot L\|x-y\|=L \Phi\|x-y\| .
\end{aligned}
$$

Hence, we get the result that $\|Q \mathcal{Q} x-Q y\| \leq L \Phi\|x-y\|$. As $L \Phi<1$, from (37), the operator $\mathbb{Q}$ is a contraction. Applying the well known Banach fixed point theorem, it follows that $\mathbb{Q}$ has a fixed point which is the unique solution of the boundary value problem (1). This completes the proof.

Next, the nonlinear contraction theorem will be used to prove a second existence and uniqueness result.

Definition 10. Let $E$ be a Banach space and let $\mathscr{A}: E \longrightarrow E$ be a mapping. The operator $\mathscr{A}$ is said to be a nonlinear contraction if there exists a continuous nondecreasing function $\Psi: \mathbb{R}^{+} \longrightarrow \mathbb{R}^{+}$such that $\Psi(0)=0$ and $\Psi(t)<t$ for all $t>0$ with the property:

$$
\|\mathscr{A} x-\mathscr{A} y\| \leq \Psi(\|x-y\|), \forall x, y \in E
$$

Lemma 11 (see [28]). Let $E$ be a Banach space and let $\mathscr{A}: E \longrightarrow E$ be a nonlinear contraction. Then, $\mathscr{A}$ has a unique fixed point in $E$.

Theorem 12. Suppose that a continuous function $f:[0, T]$ $\times \mathbb{R} \longrightarrow \mathbb{R}$ satisfies the condition: 
$\left(H_{2}\right)|f(t, x)-f(t, y)| \leq h(t)\left(|x-y| / H^{*}+|x-y|\right), \quad t \in[0$, $T], x, y \in \mathbb{R}$, where the function $h:[0, T] \longrightarrow \mathbb{R}^{+}$is continuous, and a positive constant $H^{*}$ is defined by

$$
\begin{aligned}
H^{*}= & \frac{T^{\alpha-1}}{|\Omega|} \sum_{j=1}^{m}\left|\beta_{j}\right| J^{\sigma_{j}} I_{q}^{\alpha} h\left(\eta_{j}\right) \\
& +\frac{T^{\alpha-1}}{|\Omega|} \sum_{i=1}^{n}\left|\gamma_{i}\right| I_{p_{i}}^{\mu_{i}} I_{q}^{\alpha} h\left(\xi_{i}\right)+I_{q}^{\alpha} h(T) .
\end{aligned}
$$

Then, the mixed fractional Hadamard and quantum integrals nonlocal problem (1) has a unique solution on $[0, T]$.

Proof. Let us consider the operator $\mathbb{Q}: \mathscr{C} \longrightarrow \mathscr{C}$ defined in (34) and define a continuous nondecreasing function $\Psi: \mathbb{R}^{+}$ $\longrightarrow \mathbb{R}^{+}$by

$$
\Psi(\lambda)=\frac{H^{*} \lambda}{H^{*}+\lambda}, \quad \forall \lambda \geq 0
$$

Then, we see that the function $\Psi$ satisfies $\Psi(0)=0$ and $\Psi(\lambda)<\lambda$ for all $\lambda>0$.

Next, for any $x, y \in \mathscr{C}$ and for each $t \in[0, T]$, we obtain

$$
\begin{aligned}
\mid \mathrm{Q} x(t) & -Q y(t) \mid \\
\leq & \frac{t^{\alpha-1}}{|\Omega|} \sum_{j=1}^{m}\left|\beta_{j}\right| J^{\sigma_{j}} I_{q}^{\alpha}\left|f_{x}-f_{y}\right|\left(\eta_{j}\right) \\
& +\frac{t^{\alpha-1}}{|\Omega|} \sum_{i=1}^{n}\left|\gamma_{i}\right| I_{p_{i}}^{\mu_{i}} I_{q}^{\alpha}\left|f_{x}-f_{y}\right|\left(\xi_{i}\right)+I_{q}^{\alpha}\left|f_{x}-f_{y}\right|(t) \\
\leq & \frac{T^{\alpha-1}}{|\Omega|} \sum_{j=1}^{m}\left|\beta_{j}\right| J^{\sigma_{j}} I_{q}^{\alpha}\left(h \frac{|x-y|}{H^{*}+|x-y|}\right)\left(\eta_{j}\right) \\
& +\frac{T^{\alpha-1}}{|\Omega|} \sum_{i=1}^{n}\left|\gamma_{i}\right| I_{p_{i}}^{\mu_{i}} I_{q}^{\alpha}\left(h \frac{|x-y|}{H^{*}+|x-y|}\right)\left(\xi_{i}\right) \\
& +I_{q}^{\alpha}\left(h \frac{|x-y|}{H^{*}+|x-y|}\right)(T) \leq \frac{\Psi(\|x-y\|)}{H^{*}} \\
& \cdot\left[\frac{T^{\alpha-1}}{|\Omega|} \sum_{j=1}^{m}\left|\beta_{j}\right| J^{\sigma_{j}} I_{q}^{\alpha} h\left(\eta_{j}\right)+\frac{T^{\alpha-1}}{|\Omega|} \sum_{i=1}^{n}\right. \\
& \left.\cdot\left|\gamma_{i}\right| I_{p_{i}}^{\mu_{i}} I_{q}^{\alpha} h\left(\xi_{i}\right)+I_{q}^{\alpha} h(T)\right]=\Psi(\|x-y\|),
\end{aligned}
$$

which implies that $\|Q x-Q y\| \leq \Psi(\|x-y\|)$ and also satisfies Definition 10. Therefore, $\mathbb{Q}$ is a nonlinear contraction. Thus, by applying Lemma 11 , the operator $\mathbb{Q}$ has a unique fixed point which is the unique solution of the boundary value problem (1). The proof is finished.

Next, the first existence result will be obtained by applying the following theorem.
Theorem 13 (Nonlinear alternative for single valued maps) [29]. Let $E$ be a Banach space, $C$ a closed, convex subset of $E$, $U$ be an open subset of $C$, and $0 \in U$. Suppose that $\mathscr{A}: \bar{U}$ $\longrightarrow C$ is a continuous, compact (that is, $\mathscr{A}(\bar{U})$ is a relatively compact subset of C) map. Then, either

(i) $\mathscr{A}$ has a fixed point in $\bar{U}$, or

(ii) There is a $x \in \partial U$ (the boundary of $U$ in $C$ ) and $\lambda \epsilon$ $(0,1)$ with $x=\lambda \mathscr{A}(x)$.

Theorem 14. Suppose that $f:[0, T] \times \mathbb{R} \longrightarrow \mathbb{R}$ is a nonlinear continuous function which satisfies the following conditions:

$\left(\mathrm{H}_{3}\right)$ there exists a continuous nondecreasing function $\psi:[0, \infty) \longrightarrow(0, \infty)$ and also a function $p \in C\left([0, T], \mathbb{R}^{+}\right)$ such that

$$
|f(t, x)| \leq p(t) \psi(|x|) \text { for each }(t, x) \in[0, T] \times \mathbb{R}
$$

$\left(H_{4}\right)$ there exists a positive constant $\mathrm{N}$ such that

$$
\frac{N}{\psi(N)\|p\| \Phi}>1
$$

where $\Phi$ defined by (38). Then, the problem (1) has at least one solution on $[0, T]$.

Proof. For a positive number $\rho$, we let $B_{\rho}=\{x \in \mathscr{C}:\|x\| \leq$ $\rho$ \} be a bounded ball in $\mathscr{C}$. Now, we will prove that the set $Q B_{\rho}$ is uniformly bounded. For $t \in[0, T]$, we can compute that

$$
\begin{aligned}
|Q x(t)| \leq & \frac{T^{\alpha-1}}{|\Omega|}\left[\sum_{j=1}^{m}\left|\beta_{j}\right| J^{\sigma_{j}} I_{q}^{\alpha}\left|f_{x}\right|\left(\eta_{j}\right)\right. \\
& \left.+\sum_{i=1}^{n}\left|\gamma_{i}\right| I_{p_{i}}^{\mu_{i}} I_{q}^{\alpha}\left|f_{x}\right|\left(\xi_{i}\right)\right]+I_{q}^{\alpha}\left|f_{x}\right|(T) \\
\leq & \|p\| \psi(\|x\|) \frac{T^{\alpha}}{\Gamma_{q}(\alpha+1)}\left[\frac{1}{T|\Omega|} \sum_{j=1}^{m}\left|\beta_{j}\right| \alpha^{-\sigma_{j}} \eta_{j}\right. \\
& \left.+\frac{1}{T|\Omega|} \sum_{i=1}^{n}\left|\gamma_{i}\right| \frac{\Gamma_{p_{i}}(\alpha+1)}{\Gamma_{p_{i}}\left(\alpha+\mu_{i}+1\right)} \xi_{i}^{\alpha+\mu_{i}}+1\right] \\
\leq & \|p\| \psi(\rho) \Phi
\end{aligned}
$$

which can be deduced that

$$
\|\mathbb{Q} x\| \leq\|p\| \psi(\rho) \Phi
$$

Then, the set $Q B_{\rho}$ is uniformly bounded. Next, we will show that the set $Q_{\rho}$ is equicontinuous set of $\mathscr{C}$. 
For any two points $\tau_{1}, \tau_{2} \in[0, T]$ with $\tau_{1}<\tau_{2}$ and $x \in B_{\rho}$, we have

$$
\begin{aligned}
\left|Q x\left(\tau_{2}\right)-Q x\left(\tau_{1}\right)\right| & \frac{\left|\tau_{2}^{\alpha-1}-\tau_{1}^{\alpha-1}\right|}{|\Omega|}\left|\sum_{j=1}^{m}\right| \beta_{j}\left|J^{\sigma_{j}} I_{q}^{\alpha}\right| f_{x} \mid\left(\eta_{j}\right) \\
& \left.+\sum_{i=1}^{n}\left|\gamma_{i}\right| I_{p_{i}}^{\mu_{i}} I_{q}^{\alpha}\left|f_{x}\right|\left(\xi_{i}\right)\right]+\mid \frac{1}{\Gamma_{q}(\alpha)} \int_{0}^{\tau_{1}}\left[\left(t_{2}-q s\right)_{q}^{(\alpha-1)}\right. \\
& \left.-\left(t_{1}-s\right)_{q}^{(\alpha-1)}\right] f(s, x(s)) d_{q} s+\frac{1}{\Gamma_{q}(\alpha)} \\
& \cdot \int_{\tau_{1}}^{\tau_{2}}\left(t_{2}-q s\right)_{q}^{(\alpha-1)} f(s, x(s)) d_{q} s \mid \\
\leq & \frac{\|p\| \psi(\rho)\left|\tau_{2}^{\alpha-1}-\tau_{1}^{\alpha-1}\right|}{|\Omega|} \sum_{j=1}^{m}\left|\beta_{j}\right| \frac{\alpha^{-\sigma_{j}} \eta_{j}^{\alpha}}{\Gamma_{q}(\alpha+1)} \\
& +\frac{\|p\| \psi(\rho)\left|\tau_{2}^{\alpha-1}-\tau_{1}^{\alpha-1}\right|}{|\Omega|} \sum_{i=1}^{n}\left|\gamma_{i}\right| \\
& \cdot \frac{\Gamma_{p_{i}}(\alpha+1)}{\Gamma_{p_{i}}\left(\alpha+\mu_{i}+1\right) \Gamma_{q}(\alpha+1)} \xi_{i}^{\alpha+\mu_{i}} \\
& +\frac{\|p\| \psi(\rho)}{\Gamma_{q}(\alpha+1)}\left[2\left(\tau_{2}-\tau_{1}\right)^{(\alpha)}+\left|\tau_{2}^{(\alpha)}-\tau_{1}^{(\alpha)}\right|\right] .
\end{aligned}
$$

As $\tau_{2}-\tau_{1} \longrightarrow 0$, the right hand side of the above inequality converses to zero, independently of $x \in B_{\rho}$. Then, the set $Q B_{\rho}$ is equicontinuous. Thus, we conclude that the set $Q B_{\rho}$ is relatively compact. Therefore, by the Arzel $a^{\prime}$-Ascoli theorem, the operator $\mathbb{Q}: \mathscr{C} \longrightarrow \mathscr{C}$ is completely continuous.

Finally, we show that the operator $\mathbb{Q}$ cannot be fulfilled the condition (ii) in Theorem 13. Then, we have to claim that there exists an open set $U \subset B_{\rho}$ with $x \neq \lambda Q \mathcal{Q} x$ for $\lambda \in(0,1)$ and $x \in \partial U$. Then, for each $t \in[0, T]$, we apply the computation in the first step, that is

$$
|x(t)| \leq\|p\| \psi(\|x\|) \Phi
$$

which yields inequality

$$
\frac{\|x\|}{\|p\| \psi(\|x\|) \Phi} \leq 1
$$

The condition $\left(\mathrm{H}_{4}\right)$ implies that there exists a constant $\mathrm{N}$ such that $\|x\| \neq N$. Now, we define the set

$$
U=\left\{x \in B_{\rho}:\|x\|<N\right\}
$$

From the previous results, we obtain that the operator $Q \sim \bar{U} \longrightarrow \mathscr{C}$ is continuous and completely continuous. Then, there is no $x \in \partial U$ such that $x=\lambda Q x$ for some $\lambda$ $\in(0,1)$. By applying the nonlinear alternative of the Leray-Schauder type, we get that the operator $\mathbb{Q}$ has a fixed point $x \in \bar{U}$ which is a solution of the nonlinear fractional $q$-difference equation with fractional Hadamard and quantum integral nonlocal conditions. This finishes the proof.

The next existence result is based on Krasnosel'ski i's fixed point theorem which can be used to relax the condition in Theorem 9.

Theorem 15 (Krasnosel'ski i's fixed point theorem) [30]. Let $C$ be a closed, bounded, convex, and nonempty subset of a Banach space E. Let $\mathscr{A}, \mathscr{B}$ be the operators such that (a) $\mathscr{A} x$ $+\mathscr{B} y \in C$ whenever $x, y \in C$; (b) $\mathscr{A}$ is compact and continuous; (c) $\mathscr{B}$ is a contraction mapping. Then, there exists $z \in C$ such that $z=\mathscr{A} z+\mathscr{B} z$.

Theorem 16. Assume that a continuous function $f:[0, T] \times$ $\mathbb{R} \longrightarrow \mathbb{R}$ is satisfied condition $\left(H_{1}\right)$ in Theorem 9 and is bounded as the following condition:

(i) $\left(H_{5}\right)|f(t, x)| \leq \kappa(t), \quad \forall(t, x) \in[0, T] \times \mathbb{R}$, and $\kappa \in C$ $\left([0, T], \mathbb{R}^{+}\right)$.

If inequality

$$
\frac{L T^{\alpha}}{\Gamma_{q}(\alpha+1)}<1
$$

holds, then the nonlocal problem (1) has at least one solution on $[0, T]$.

Proof. Now, we define $\sup \{|\kappa(t)|: t \in[0, T]\}=\|\kappa\|$ and choose a positive constant $\bar{r}$ such that

$$
\bar{r} \geq\|\kappa\| \Phi
$$

where $\Phi$ is defined by (38), to be a radius of the ball $B_{\bar{r}}=\{x \in \mathscr{C}:\|x\| \leq \bar{r}\}$. Furthermore, we set the operators $Q_{1}$ and $Q_{2}$ on $B_{\bar{r}}$ as $\mathscr{A}$ and $\mathscr{B}$ in Theorem 15, respectively, by

$$
\begin{aligned}
\mathcal{Q}_{1} x(t)= & \frac{t^{\alpha-1}}{\Omega}\left[\sum_{j=1}^{m} \beta_{j} J^{\sigma_{j}} I_{q}^{\alpha} f_{x}\left(\eta_{j}\right)\right. \\
& \left.-\sum_{i=1}^{n} \gamma_{i} I_{p_{i}}^{\mu_{i}} I_{q}^{\alpha} f_{x}\left(\xi_{i}\right)\right], t \in[0, T], \\
\mathcal{Q}_{2} x(t)= & I_{q}^{\alpha} f_{x}(t) t \in[0, T] .
\end{aligned}
$$


The combination of two operators shows $Q_{1}+Q_{2}=Q$. We have

$$
\begin{aligned}
\left\|Q_{1} x+\mathcal{Q}_{2} y\right\| \leq & \|\kappa\|\left[\frac{T^{\alpha-1}}{|\Omega|} \sum_{j=1}^{m}\left|\beta_{j}\right| \frac{\alpha^{-\sigma_{j}} \eta_{j}^{\alpha}}{\Gamma_{q}(\alpha+1)}\right. \\
& +\frac{T^{\alpha-1}}{|\Omega|} \sum_{i=1}^{n}\left|\gamma_{i}\right| \frac{\Gamma_{p_{i}}(\alpha+1)}{\Gamma_{p_{i}}\left(\alpha+\mu_{i}+1\right) \Gamma_{q}(\alpha+1)} \xi_{i}^{\alpha+\mu_{i}} \\
& \left.+\frac{T^{\alpha}}{\Gamma_{q}(\alpha+1)}\right]=\|\kappa\| \Phi \leq \bar{r} .
\end{aligned}
$$

Therefore, we have $Q_{1} x+Q_{2} y \in B_{\bar{r}}$, and thus condition (a) of Theorem 15 is satisfied. Since the function $f$ is fulfilled by condition $\left(H_{1}\right)$ in Theorem 9 , then the operator $Q_{2}$ is a contraction mapping with inequality (57).

Finally, we will show that the operator $Q_{1}$ should satisfy condition $(b)$ in Theorem 15 . Using the continuity of $f$, we can show that the operator $Q_{1}$ is continuous. The uniformly boundedness of the set $Q_{1} B_{\bar{r}}$ can be shown by

$\left\|Q_{1} x\right\| \leq \frac{\|\kappa\| T^{\alpha-1}}{|\Omega| \Gamma_{q}(\alpha+1)}\left[\sum_{j=1}^{m}\left|\beta_{j}\right| \alpha^{-\sigma_{j}} \eta_{j}^{\alpha}+\sum_{i=1}^{n}\left|\gamma_{i}\right| \frac{\Gamma_{p_{i}}(\alpha+1)}{\Gamma_{p_{i}}\left(\alpha+\mu_{i}+1\right)} \xi_{i}^{\alpha+\mu_{i}}\right]$.

To prove $Q_{1} B_{\bar{r}}$ is equicontinuous set, we let two points $t_{1}, t_{2} \in[0, T], t_{2}<t_{1}$. For any $x \in B_{\bar{r}}$, we have

$$
\begin{aligned}
& \left|\mathcal{Q}_{1} x\left(t_{1}\right)-Q_{1} x\left(t_{2}\right)\right| \\
& \leq\|\kappa\| \frac{\left|t_{1}^{\alpha-1}-t_{2}^{\alpha-1}\right|}{|\Omega|} \sum_{j=1}^{m}\left|\beta_{j}\right| \frac{\alpha^{-\sigma_{j}} \eta_{j}^{\alpha}}{\Gamma_{q}(\alpha+1)} \\
& \quad+\|\kappa\| \frac{\left|t_{1}^{\alpha-1}-t_{2}^{\alpha-1}\right|}{|\Omega|} \sum_{i=1}^{n}\left|\gamma_{i}\right| \frac{\Gamma_{p_{i}}(\alpha+1)}{\Gamma_{p_{i}}\left(\alpha+\mu_{i}+1\right) \Gamma_{q}(\alpha+1)} \xi_{i}^{\alpha+\mu_{i}},
\end{aligned}
$$

which converses to zero independently of $x$ as $\left|t_{1}-t_{2}\right| \longrightarrow 0$. So, $Q_{1} B_{\bar{r}}$ is an equicontinuous set. Therefore, $Q_{1} B_{\bar{r}}$ is a relative compact and by the Arzelá-Ascoli theorem, $Q_{1}$ is compact on $B_{\bar{r}}$. Thus, the assumptions $(a),(b)$, and $(c)$ of Krasnosel'ski i's fixed point theorem are satisfied. Then, the nonlinear fractional $q$-difference equation with fractional Hadamard and quantum integral nonlocal conditions (1) has at least one solution on $[0, T]$. The proof is completed.

Remark 17. The interchanging of operators $\mathbb{Q}_{1}$ and $\mathbb{Q}_{2}$ gives another result by replacing inequality (57) by the following condition:

$\frac{L T^{\alpha-1}}{|\Omega| \Gamma_{q}(\alpha+1)}\left[\sum_{j=1}^{m}\left|\beta_{j}\right| \alpha^{-\sigma_{j}} \eta_{j}^{\alpha}+\sum_{i=1}^{n}\left|\gamma_{i}\right| \frac{\Gamma_{p_{i}}(\alpha+1)}{\Gamma_{p_{i}}\left(\alpha+\mu_{i}+1\right)} \xi_{i}^{\alpha+\mu_{i}}\right]<1$.

\section{Examples}

Example 18. Consider the nonlinear fractional $q$-difference equation with fractional Hadamard and quantum integral nonlocal conditions of the form:

$$
\left(\begin{array}{l}
D_{1 / 2}^{3 / 2} x(t)=f(t, x(t)), t \in(0,2), \\
x(0)=0, \frac{3}{8} I_{1 / 6}^{1 / 2} x\left(\frac{1}{4}\right)+\frac{2}{5} I_{1 / 3}^{3 / 2} x\left(\frac{1}{2}\right)+\frac{1}{9} I_{1 / 2}^{5 / 2} x\left(\frac{3}{2}\right) \\
=\frac{1}{3} J^{1 / 3} x\left(\frac{1}{5}\right)+\frac{4}{9} J^{2 / 3} x\left(\frac{3}{5}\right)+\frac{7}{12} J^{4 / 3} x\left(\frac{7}{5}\right)+\frac{8}{15} J^{5 / 3} x\left(\frac{9}{5}\right) .
\end{array}\right.
$$

Here, $\alpha=3 / 2, q=1 / 2, T=2, \gamma_{1}=3 / 8, \gamma_{2}=2 / 5, \gamma_{3}=1 / 9, \mu_{1}$ $=1 / 2, \mu_{2}=3 / 2, \mu_{3}=5 / 2, p_{1}=1 / 6, p_{2}=1 / 3, p_{3}=1 / 2, \xi_{1}=1 /$ $4, \xi_{2}=1 / 2, \xi_{3}=3 / 2, n=3, \beta_{1}=1 / 3, \beta_{2}=4 / 9, \beta_{3}=7 / 12, \beta_{4}$ $=8 / 15, \sigma_{1}=1 / 3, \sigma_{2}=2 / 3, \sigma_{3}=4 / 3, \sigma_{4}=5 / 3, \eta_{1}=1 / 5, \eta_{2}=3$ $15, \eta_{3}=7 / 5, \eta_{4}=9 / 5, m=4$. Then, we can compute constants as $|\Omega| \approx 2.51852$ and $\Phi \approx 3.27524$.

(i) Let the nonlinear function $f$ be defined by

$$
f(t, x)=\frac{e^{-\cos ^{2} t}}{(t+2)^{3}}\left(\frac{x^{2}+2|x|}{1+|x|}\right)+\frac{t^{2}}{4}+1 .
$$

Then, by direct computation, we get $|f(t, x)-f(t, y)| \leq$ $(1 / 4)|x-y|$, which satisfies condition $\left(H_{1}\right)$ in Theorem 9 with $L=1 / 4$. Therefore, we have

$$
L \Phi \approx 0.81881<1
$$

By the conclusion of Theorem 9, the boundary value problem (64) with (65) has a unique solution on $[0,2]$.

(ii) Consider now the function $f$ by

$$
f(t, x)=\frac{1}{(t+2)^{3}}\left(\frac{x^{18}}{x^{16}+1}+1\right) \text {. }
$$

Then, we can see that

$$
|f(t, x)|=\left|\frac{1}{(t+2)^{3}}\left(\frac{x^{18}}{x^{16}+1}+1\right)\right| \leq \frac{1}{(t+2)^{3}}\left(x^{2}+1\right)
$$

Setting $p(t)=1 /(t+2)^{3}$ and $\psi(x)=x^{2}+1$, we have $\|p\|$ $=1 / 8$, and there exists a constant $N \in(0.52019,1.92238)$ satisfying inequality in $\left(\mathrm{H}_{4}\right)$. Hence, all assumptions in Theorem 14 are completed. Thus, the problem (64) with (67) has at least one solution on $[0,2]$.

(iii) If the function $f$ is

$$
f(t, x)=\frac{\sin ^{2} t}{m}\left(\frac{|x|}{|x|+1}\right)+\frac{1}{4}, \quad m \in \mathbb{R}^{+},
$$

then, we have $|f(t, x)-f(t, y)| \leq(1 / m)|x-y|$ with $L=1 / m$. If $m \leq \Phi \approx 3.27524$, then Theorem 9 cannot be used to apply for the problem (64) with (69). For example, if $m=2$, then 
$L \Phi \approx 1.63762>1$. But the inequality in Remark 17 is satisfied as

$$
\begin{aligned}
& \frac{1}{2} \cdot \frac{T^{\alpha-1}}{|\Omega| \Gamma_{q}(\alpha+1)}\left[\sum_{j=1}^{m}\left|\beta_{j}\right| \alpha^{-\sigma_{j}} \eta_{j}^{\alpha}+\sum_{i=1}^{n}\left|\gamma_{i}\right| \frac{\Gamma_{p_{i}}(\alpha+1)}{\Gamma_{p_{i}}\left(\alpha+\mu_{i}+1\right)} \xi_{i}^{\alpha+\mu_{i}}\right] \\
& \quad=0.44979<1 .
\end{aligned}
$$

Hence, by applying Theorem 16 and Remark 17, the problem (64) with (69) has at least one solution on $[0,2]$.

\section{Conclusion}

We investigated the existence and uniqueness of solutions for a nonlocal boundary value problem involving a $q$-difference equation, supplemented with a new type of boundary condition, including both Hadamard fractional and quantum integrals. In our first two results, we establish the existence and uniqueness of solutions by using Banach's fixed point theorem and a fixed point theorem for nonlinear contractions due to Boyd and Wong. Then, we used the Leray-Schauder nonlinear alternative and Krasnosel'ski i's fixed point theorem to derive two existence results. Examples are also presented to illustrate our results. It is worthwhile to point out that the results presented in this paper are new and significantly contribute to the existing literature on the topic.

\section{Data Availability}

No data were used to support this study.

\section{Conflicts of Interest}

The authors declare that there are no conflicts of interest regarding the publication of this paper.

\section{Acknowledgments}

This research was funded by King Mongkut's University of Technology North Bangkok. Contract no. KMUTNB-61KNOW-031.

\section{References}

[1] A. A. Kilbas, H. M. Srivastava, and J. J. Trujillo, Theory and Applications of Fractional Differential Equations, NorthHolland Mathematics Studies, Elsevier Science B.V, Amsterdam, 2006.

[2] B. Ahmad and S. K. Ntouyas, "Boundary value problems of Hadamard-type fractional differential equations and inclusions with nonlocal conditions," Vietnam Journal of Mathematics, vol. 45, no. 3, pp. 409-423, 2017.

[3] J. Mao, Z. Zhao, and C. Wang, "The unique positive solution for singular Hadamard fractional boundary value problems," Journal of Function Spaces, vol. 2019, Article ID 5923490, 6 pages, 2019.

[4] G. Wang, K. Pei, R. P. Agarwal, L. Zhang, and B. Ahmad, "Nonlocal Hadamard fractional boundary value problem with Hadamard integral and discrete boundary conditions on a half-line," Journal of Computational and Applied Mathematics, vol. 343, pp. 230-239, 2018.

[5] S. Etemad, S. Rezapour, and F. M. Sakar, "On a fractional Caputo-Hadamard problem with boundary value conditions via different orders of the Hadamard fractional operators," Advances in Difference Equations, vol. 2020, no. 1, 2020.

[6] B. Ahmad and S. K. Ntouyas, "A fully Hadamard type integral boundary value problem of a coupled system of fractional differential equations," Fractional Calculus and Applied Analysis, vol. 17, pp. 348-360, 2014.

[7] S. K. Ntouyas, J. Tariboon, and C. Thaiprayoon, "Nonlocal boundary value problems for Riemann-Liouville fractional differential inclusions with Hadamard fractional integral boundary conditions," Taiwanese Journal of Mathematics, vol. 20, no. 1, pp. 91-107, 2016.

[8] S. Asawasamrit, S. K. Ntouyas, J. Tariboon, and W. Nithiarayaphaks, "Coupled systems of sequential Caputo and Hadamard fractional differential equations with coupled separated boundary conditions," Symmetry, vol. 10, no. 12, p. 701, 2018.

[9] B. Ahmad, A. Alsaedi, S. K. Ntouyas, and J. Tariboon, Hadamard-Type Fractional Differential Equations, Inclusions and Inequalities, Springer, Cham, Switzerland, 2017.

[10] J. Tariboon, S. K. Ntouyas, and W. Sudsutad, "Nonlocal Hadamard fractional integral conditions for nonlinear RiemannLiouville fractional differential equations," Boundary Value Problems, vol. 2014, no. 1, 2014.

[11] W. Saengthong, E. Thailert, and S. K. Ntouyas, "Existence and uniqueness of solutions for system of Hilfer-Hadamard sequential fractional differential equations with two point boundary conditions," Advances in Difference Equations, vol. 2019, no. 1, 2019.

[12] B. Ahmad and S. K. Ntouyas, "Boundary value problems for qdifference equations and inclusions with nonlocal and integral boundary conditions," Mathematical Modelling and Analysis, vol. 19, no. 5, pp. 647-663, 2014.

[13] C. Yang, "Positive solutions for a three-point boundary value problem of fractional q-difference equations," Symmetry, vol. 10, no. 9, p. 358, 2018.

[14] C. Guo, J. Guo, S. Kang, and H. Li, "Existence and uniqueness of positive solution for nonlinear fractional Q-difference equation with integral boundary conditions," Journal of Applied Analysis \& Computation, vol. 10, no. 1, pp. 153-164, 2020.

[15] R. Ouncharoen, N. Patanarapeelert, and T. Sitthiwirattham, "Nonlocal q-symmetric integral boundary value problem for sequential q-symmetric integrodifference equations," Mathematics, vol. 6, no. 11, p. 218, 2018.

[16] C. Zhai and J. Ren, "Positive and negative solutions of a boundary value problem for a fractional q-difference equation," Advances in Difference Equations, vol. 2017, no. 1, 2017.

[17] J. Ren, "Nonlocal q-fractional boundary value problem with Stieltjes integral conditions," Nonlinear Analysis: Modelling and Control, vol. 24, pp. 582-602, 2019.

[18] J. Ren and C. Zhai, "Characteristic of unique positive solution for a fractional q-difference equation with multistrip boundary conditions," Mathematical Communications, vol. 24, pp. 181192, 2019.

[19] J. Ren and C. Zhai, “A fractional q\$q \$-difference equation with integral boundary conditions and comparison theorem," International Journal of Nonlinear Sciences and Numerical Simulation, vol. 18, no. 7-8, pp. 575-583, 2017. 
[20] J. Ren and C. Zhai, "Unique solutions for fractional q-difference boundary value problems via a fixed point method," Bulletin of the Malaysian Mathematical Sciences Society, vol. 42, no. 4, pp. 1507-1521, 2019.

[21] C. Zhai and J. Ren, "The unique solution for a fractional q -difference equation with three-point boundary conditions," Indagationes Mathematicae, vol. 29, pp. 948-961, 2018.

[22] V. Kac and P. Cheung, Quantum Calculus, Springer, New York, NY, USA, 2002.

[23] W. A. Al-Salam, "Some fractional q-integrals and q-derivatives," Proceedings of the Edinburgh Mathematical Society, vol. 15, no. 2, pp. 135-140, 1966.

[24] R. P. Agarwal, "Certain fractional q-integrals and q-derivatives," Proceedings of the Cambridge Philosophical Society, vol. 66, no. 2, pp. 365-370, 1969.

[25] M. H. Annaby and Z. S. Mansour, q-Fractional Calculus and Equations, Lecture Notes in Mathematics 2056, Springer-Verlag, Berlin, 2012.

[26] R. A. C. Ferreira, "Nontrivial solutions for fractional qdifference boundary value problems," Electronic Journal of Qualitative Theory of Differential Equations, vol. 2010, no. 70, pp. 1-10, 2010.

[27] S. Suantai, S. K. Ntouyas, S. Asawasamrit, and J. Tariboon, "A coupled system of fractional q-integro-difference equations with nonlocal fractional q-integral boundary conditions," Advances in Difference Equations, vol. 2015, no. 1, 2015.

[28] D. W. Boyd and J. S. W. Wong, "On nonlinear contractions," Proceedings of the American Mathematical Society, vol. 20, no. 2, pp. 458-464, 1969.

[29] A. Granas and J. Dugundji, Fixed Point Theory, Springer-Verlag, New York, 2003.

[30] M. A. Krasnosel'ski1, "Two remarks on the method of successive approximations," Uspekhi Matematicheskikh Nauk, vol. 10, pp. 123-127, 1955. 